\title{
A Concise and Enantioselective Synthesis of a C-6 O-Acyl Side Chain Equivalent of Zaragozic Acid A
}

\author{
Seiichi Nakamura, Jun Inagaki, Junichi Kitaguchi, Kazuya Tatani, and Shunichi Hashimoto* \\ Graduate School of Pharmaceutical Sciences, Hokkaido University, Sapporo 060-0812, Japan. \\ Received April 26, 1999; accepted May 31, 1999
}

\begin{abstract}
An optically pure $C-6 O$-acyl side chain equivalent of zaragozic acid $A,(2 E, 4 S, 6 S)$-4,6-dimethyl-2-octenoic acid, which features a 1,3-syn-dimethyl-substituted carbon chain, has been readily synthesized from $(S)$-2methylbutanal using a combination of the Evans aldol reaction and the Ireland deoxygenation method.
\end{abstract}

Key words zaragozic acid A; acyl side chain; Evans aldol reaction; Ireland deoxygenation method

Zaragozic acids and squalestatins, recently isolated from various fungal cultures by respective researchers at Merck and Glaxo, have been shown to be picomolar competitive inhibitors of squalene synthase. ${ }^{1)}$ In addition to their therapeutic potential for the treatment of hypercholesterolemia, these natural products possess a novel, densely oxygenated 4,6,7trihydroxy-2,8-dioxabicyclo[3.2.1] octane-3,4,5-tricarboxylic acid core, differing only in regard to the nature of the $\mathrm{C} 1$ alkyl and $\mathrm{C} 6 \mathrm{O}$-acyl side chains. It is therefore not surprising that the zaragozic acids (squalestatins) have elicited considerable attention from numerous synthetic chemists. ${ }^{2,3)}$ The Nicolaou $^{4)}$ and Heathcock ${ }^{5)}$ groups have accomplished the total synthesis of zaragozic acid A (squalestatin S1), while efforts of the groups of Carreira, ${ }^{6)}$ Evans, ${ }^{7)}$ and Armstrong, ${ }^{8)}$ as well as ours, ${ }^{9)}$ have culminated in the total synthesis of zaragozic acid $\mathrm{C}$. Accordingly, our attention has now been turned to the total synthesis of zaragozic acid A. Herein we wish to report the easy and efficient access to $(2 E, 4 S, 6 S)-4,6-$ dimethyl-2-octenoic acid (1), the $\mathrm{C}-6 \quad O$-acyl side chain equivalent. $^{4,5 a, 10)}$

The $\alpha, \beta$-unsaturated acid 1 was reported to be elaborated from $(2 S, 4 S)$-2,4-dimethyl-1-hexanol (2) via oxidation and Wittig-type olefination. ${ }^{5 a, 10 b)}$ Thus, alcohol 2 became a focal intermediate which features a minimum carbon chain with skip 1,3-syn-dimethyl stereogenic centers. Of a variety of approaches considered for the enantioselective synthesis of $\mathbf{2}$, a route proceeding through the asymmetric alkylation of a chiral propionate-derived enolate with readily available $(S)-2-$ methyl-1-iodobutane (3) was deemed highly attractive from the standpoint of conciseness and convergency. In this context, it has been well documented that Evans' chiral oxazolidinone-derived carboximide enolates ${ }^{11)}$ do not possess sufficient nucleophilicity toward $\beta$-branched alkyl halides such as 3. $^{12)}$ Although Decicco and Grover recently demonstrated that the enolates did react with $\beta$-branched alkyl triflates to give all four possible diastereomers with excellent stereocontrol, a large excess of the electrophile ( $25 \mathrm{eq})$ was required in the reaction. ${ }^{13)}$ In the synthesis of $(+)$-bourgeanic acid, White and Johnson reported that asymmetric alkylation of an enolate derived from $(S)$-prolinol $N$-propionamide ${ }^{14)}$ with 3 gave a 17:1 mixture of the adducts with the desired syn product as the major constituent, which, after saponification, was purified to optical homogeneity by recrystallization of the salt of the carboxylic acid and cinchonidine. ${ }^{15)}$ In the synthesis of zaragozic acid A, Nicolaou and co-workers synthesized 2 via $(2 S, 4 S)$-2,4-dimethyl-1-hexanal (4) of $92 \% \mathrm{de}^{4)}$

* To whom correspondence should be addressed. which was obtained by the alkylation of Enders' chiral hydrazone enolate ${ }^{16)}$ with 3 . In the same context, Heathcock and co-workers who were cognizant of the shortcoming of Evans' chiral oxazolidinone-derived carboximide enolates, as described above, addressed an alternative alkylation and found access to optically pure 2 ; methylation of the imide prepared from Evans' chiral oxazolidinone and (S)-4-methylhexanoic acid, derived from 3 , provided the diastereomers in a $17: 1$ ratio of syn to anti isomers, from which the syn isomer was separated by flash chromatography. ${ }^{5 a)}$ Thus, the asymmetric alkylation approach to optically pure 1, employing well-established chiral auxiliaries, has suffered from tedious separation of the diastereomers. ${ }^{17)}$

Departing from the alkylation route, we then directed our attention to an approach capitalizing on the aldol reaction ${ }^{18)}$ between chiral $N$-propionyl-2-oxazolidinone 5 and $(S)$-2methylbutanal (6), ${ }^{19)}$ followed by deoxygenation, ${ }^{20)}$ because the Evans aldol reaction provides the most reliable means for the controlled creation of vicinal syn stereogenic centers, irrespective of the absolute configuration of $\alpha$-branched aldehydes. ${ }^{21)}$ Indeed, condensation of the boron enolate derived from 5 with 6 provided the crystalline syn aldol adduct 7 as a single diastereomer in $78 \%$ yield, the homochirality of which was confirmed by $500 \mathrm{MHz}{ }^{1} \mathrm{H}$ - and $100 \mathrm{MHz}{ }^{13} \mathrm{C}-\mathrm{NMR}$. Removal of the oxazolidinone auxiliary from 7 by reduction with lithium borohydride, ${ }^{22)}$ followed by selective silylation of the resultant diol, furnished the secondary alcohol 8 in $84 \%$ yield. While a number of methods for deoxygenation were reported, ${ }^{23)}$ we initially explored a combination of mesylate formation and reduction with lithium aluminum hydride. ${ }^{24)}$ However, reduction of the mesylate was found not to take place even under harsh conditions, which might be accounted for by steric hindrance due to a syn/syn relationship

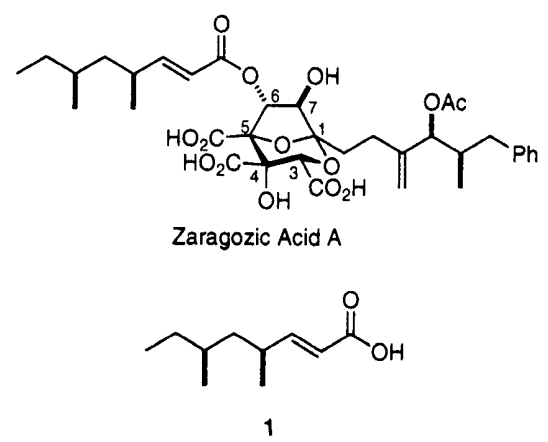

Fig. 1. Zaragozic Acid A and C-6 O-Acyl Side Chain Equivalent 1 

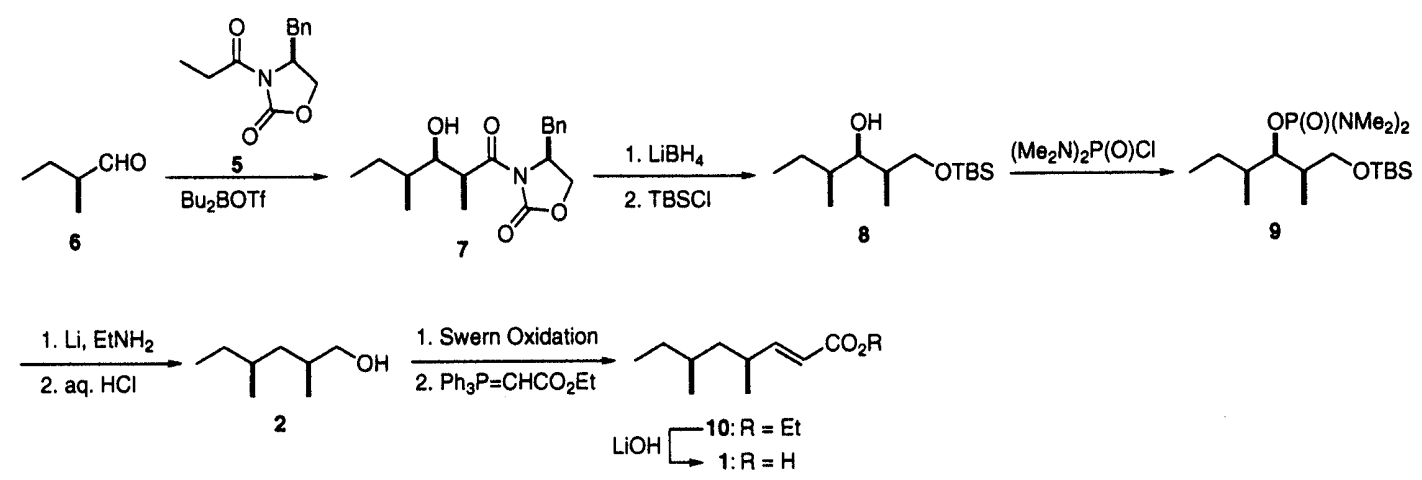

Chart 1

in the $\mathrm{C} 2-\mathrm{C} 4$ stereotriad. We then chose the Ireland deoxygenation procedure ${ }^{25)}$ in preference to the Barton-McCombie reaction, ${ }^{26)}$ because the latter method has demonstrated the formation of toxic tin byproducts difficult to remove completely from the product. Treatment of the alcohol 8 with $n$ butyllithium, followed by condensation with $N, N, N^{\prime}, N^{\prime}$ tetramethylphosphorodiamidic chloride, gave the corresponding phosphorodiamidate 9 in $80 \%$ yield, which was found to be more stable than the corresponding diethyl phosphate and is easily handled. The reduction of 9 with lithium in ethylamine at $0{ }^{\circ} \mathrm{C}$ proceeded cleanly to give a deoxygenated product, which, upon desilylation, produced the alcohol $2,[\alpha]_{\mathrm{D}}^{27}-4.56^{\circ}\left(c=1.64, \mathrm{CHCl}_{3}\right)\left[\right.$ lit. $\left.^{10 b}\right)[\alpha]_{\mathrm{D}}^{27}-4.5^{\circ}$ $\left.\left(c=1.60, \mathrm{CHCl}_{3}\right)\right]$, in $81 \%$ overall yield. Oxidation of 2 under standard Swern conditions, followed by immediate condensation with $\mathrm{Ph}_{3} \mathrm{P}=\mathrm{CHCO}_{2} \mathrm{Et}$, afforded exclusively the (E)- $\alpha, \beta$-unsaturated ester $\mathbf{1 0}$ in $84 \%$ yield. Finally, the saponification of 10 furnished the target acid $1,[\alpha]_{\mathrm{D}}^{24}+58.6^{\circ}$ (neat) $\left[\right.$ lit. $^{27)}[\alpha]_{\mathrm{D}}^{19}+55^{\circ}$ (neat)], in $98 \%$ yield, which exhibited identical spectroscopic data with those reported for a sample by the degradation of zaragozic acid A.

In summary, we have achieved the synthesis of an optically pure C-6 $O$-acyl side chain equivalent of zaragozic acid A with an overall yield of $35 \%$ for the nine-step sequence. The present protocol, employing a combination of an Evans aldol reaction and an Ireland deoxygenation method, has the advantage of providing a practical entry to 1,3-dimethyl-substituted carbon chains with virtually complete enantio- and diastereocontrol, and thus represents a potent alternative to an asymmetric alkylation strategy. ${ }^{17}$ )

\section{Experimental}

Melting points were determined on a Büchi 535 digital melting point apparatus and are uncorrected. NMR spectra were measured with JEOL JNMEX270 $\left({ }^{13} \mathrm{C}\right.$ at $\left.67.8 \mathrm{MHz}\right)$, JEOL JNM-AL400 $\left({ }^{13} \mathrm{C}\right.$ at $\left.100 \mathrm{MHz}\right)$ or Bruker ARX-500 spectrometers $\left({ }^{1} \mathrm{H}\right.$ at $\left.500 \mathrm{MHz}\right)$, with tetramethylsilane $\left(\delta 0.0,{ }^{1} \mathrm{H}\right)$ or chloroform- $d_{1}\left(\delta 77.0,{ }^{13} \mathrm{C}\right)$ as internal standards. Infrared spectra were recorded on a Jasco FT/IR-5300 spectrometer. Optical rotations were measured on a Jasco DIP-370 digital polarimeter. Electron impact (EI) mass spectra were obtained on a JEOL DX-303 spectrometer, operating with an ionization energy of $70 \mathrm{eV}$. FAB-MS were obtained on a JEOL JMS-HX110 spectrometer. Column chromatography was performed on Merck silica gel 60 (70--230 mesh). Bulb-to-bulb distillation was performed using a Büchi Kugelrohr apparatus, and the oven temperature is recorded as the boiling point. Di- $n$-butylboron triflate ${ }^{28)}$ and $(S)$-4-benzyl-3-propionyl-2-oxazolidinone $(5)^{18 b)}$ were prepared according to literature procedures.

(2'S,3' $\left.R, 4 S, 4^{\prime} S\right)$-3-3'-Hydroxy-2', $4^{\prime}$-dimethylhexanoyl-4-benzyl-2-oxazolidinone (7) To a stirred solution of $5(12.4 \mathrm{~g}, 53 \mathrm{mmol})$ in $\mathrm{CH}_{2} \mathrm{Cl}_{2}$ $(130 \mathrm{ml})$ at $0^{\circ} \mathrm{C}$ was added di- $n$-butylboron triflate $(18.0 \mathrm{ml}, 71 \mathrm{mmol})$, followed by triethylamine $(12.0 \mathrm{ml}, 86 \mathrm{mmol})$. After $0.5 \mathrm{~h}$ of stirring at $0^{\circ} \mathrm{C}$, the mixture was cooled to $-78^{\circ} \mathrm{C}$ and a solution of $(S)$-2-methylbutanal (6) $(5.9$ $\mathrm{g}, 69 \mathrm{mmol})$ in $\mathrm{CH}_{2} \mathrm{Cl}_{2}(15 \mathrm{ml})$ was added. The mixture was stirred at -78 ${ }^{\circ} \mathrm{C}$ for $1 \mathrm{~h}$, and then at $0^{\circ} \mathrm{C}$ for another $2 \mathrm{~h}$. The reaction was quenched with $\mathrm{pH} 7$ phosphate buffer $(60 \mathrm{ml})$, and the mixture was diluted with $\mathrm{MeOH}$ $(100 \mathrm{ml})$. A 2: 1 mixture of $\mathrm{MeOH}-30 \%$ aqueous $\mathrm{H}_{2} \mathrm{O}_{2}(200 \mathrm{ml})$ was added at $0^{\circ} \mathrm{C}$, and the whole was stirred vigorously for $1 \mathrm{~h}$. After the volatile elements were removed in vacuo, the residue was diluted with EtOAc $(60 \mathrm{ml})$ and the layers were separated. The organic layer was washed successively with saturated aqueous $\mathrm{NaHCO}_{3}(40 \mathrm{ml}), 10 \%$ aqueous $\mathrm{Na}_{2} \mathrm{~S}_{2} \mathrm{O}_{3}(40 \mathrm{ml})$ and brine $(2 \times 10 \mathrm{ml})$, then dried over anhydrous $\mathrm{Na}_{2} \mathrm{SO}_{4}$. Filtration and evaporation in vacuo furnished the crude product $(18.1 \mathrm{~g})$, which was purified by column chromatography (silica gel $500 \mathrm{~g}, 5: 1$ hexane-EtOAc) to give aldol adduct $7(13.2 \mathrm{~g}, 78 \%)$ as colorless needles, $\mathrm{mp} 90.0-90.5^{\circ} \mathrm{C}$ (hexane), $[\alpha]_{\mathrm{D}}^{27}+43.1^{\circ}\left(c=1.07, \mathrm{CHCl}_{3}\right)$. IR (Nujol) $\mathrm{cm}^{-1}: 3522,2924,1777,1692$, 1456, 1200. 'H-NMR $\left(\mathrm{CDCl}_{3}\right) \delta: 0.91\left(3 \mathrm{H}, \mathrm{t}, J=7.3 \mathrm{~Hz}, \mathrm{CH}_{3} \mathrm{CH}_{2}\right), 0.98$ $\left(3 \mathrm{H}, \mathrm{d}, J=6.5 \mathrm{~Hz}, \mathrm{CHCH}_{3}\right), 1.14\left(1 \mathrm{H}, \mathrm{m}, \mathrm{CH}_{3} \mathrm{CH}_{2}\right), 1.27(3 \mathrm{H}, \mathrm{d}, J=7.0 \mathrm{~Hz}$, $\left.\mathrm{CHC}_{3}\right), 1.43-1.56\left(2 \mathrm{H}, \mathrm{m}, \mathrm{C}_{2} \mathrm{CH}\right), 2.62(1 \mathrm{H}, \mathrm{br} \mathrm{s}, \mathrm{OH}), 2.79(1 \mathrm{H}, \mathrm{dd}$, $\left.J=9.5,13.4 \mathrm{~Hz}, \mathrm{PhCH}_{2}\right), 3.26\left(1 \mathrm{H}, \mathrm{dd}, J=3.3,13.4 \mathrm{~Hz}, \mathrm{PhC}_{2}\right), 3.69(1 \mathrm{H}$, $\mathrm{m}, \mathrm{CHOH}), 3.99(1 \mathrm{H}, \mathrm{dq}, J=3.7,7.0 \mathrm{~Hz}, \mathrm{C} \underline{\mathrm{HCO}}), 4.19(1 \mathrm{H}, \mathrm{dd}, J=3.0,9.0$ $\left.\mathrm{Hz}, \underline{\mathrm{C}}_{2} \mathrm{O}\right), 4.23\left(1 \mathrm{H}, \mathrm{dd}, J=7.6,9.0 \mathrm{~Hz}, \mathrm{C}_{2} \mathrm{O}\right), 4.70(1 \mathrm{H}, \mathrm{dddd}, J=3.0$, 3.3, 7.6, $9.5 \mathrm{~Hz}, \mathrm{C} \underline{\mathrm{HN}}), 7.21(2 \mathrm{H}, \mathrm{m}, \mathrm{Ar} \underline{\mathrm{H}}), 7.28(1 \mathrm{H}, \mathrm{m}, \operatorname{Ar} \underline{\mathrm{H}}), 7.34(2 \mathrm{H}, \mathrm{m}$, ArH $){ }^{13} \mathrm{C}$-NMR $\left(\mathrm{CDCl}_{3}\right) \delta: 11.2\left(\mathrm{CH}_{3} \times 2\right), 14.5\left(\mathrm{CH}_{3}\right), 25.6\left(\mathrm{CH}_{2}\right), 37.1$ $(\mathrm{CH}), 37.7\left(\mathrm{CH}_{2}\right), 39.8(\mathrm{CH}), 55.1(\mathrm{CH}), 66.1\left(\mathrm{CH}_{2}\right), 75.0(\mathrm{CH}), 127.4$ $(\mathrm{CH}), 128.9(\mathrm{CH}), 129.4(\mathrm{CH}), 135.0(\mathrm{C}), 152.8(\mathrm{C}=\mathrm{O}), 177.5(\mathrm{C}=\mathrm{O})$. EIMS $m / z$ (rel. int. \%): $319\left(\mathrm{M}^{+}, 10\right), 301$ (8.3), 262 (22), 244 (33), 178 (100), 143 (26). HR-EI-MS $m / z: 319.1757$ (Calcd for $\mathrm{C}_{18} \mathrm{H}_{25} \mathrm{NO}_{4}: 319.1784$ ). Anal. Calcd for $\mathrm{C}_{18} \mathrm{H}_{25} \mathrm{NO}_{4}$ : C, 67.69; H, 7.89; N, 4.38. Found: C, 67.85; H, 8.03; $\mathrm{N}, 4.32$

(2S,3R,4S)-1-(tert-Butyldimethylsilyloxy)-2,4-dimethylhexan-3-ol (8) A $2 \mathrm{M}$ solution of lithium borohydride in tetrahydrofuran (THF) $(22 \mathrm{ml}, 44$ $\mathrm{mmol})$ was added to a solution of aldol adduct $7(11.9 \mathrm{~g}, 37 \mathrm{mmol})$ in THF $(150 \mathrm{ml})$-water $(0.8 \mathrm{ml}, 44 \mathrm{mmol})$ at $0^{\circ} \mathrm{C}$. After being stirred at $0^{\circ} \mathrm{C}$ for $1 \mathrm{~h}$, the reaction was quenched with $0.5 \mathrm{~N}$ aqueous potassium sodium tartrate $(160 \mathrm{ml})$ at $0{ }^{\circ} \mathrm{C}$, followed by stirring at room temperature for $1 \mathrm{~h}$. After the volatile elements were removed in vacuo, the whole was extracted with EtOAc $(3 \times 60 \mathrm{ml})$. The combined organic extracts were washed with brine $(2 \times 20 \mathrm{ml})$ and dried over anhydrous $\mathrm{Na}_{2} \mathrm{SO}_{4}$. Filtration and evaporation in vacuo furnished the crude product $(11.2 \mathrm{~g})$, which was used without further purification for the next reaction. Imidazole $(5.1 \mathrm{~g}, 75 \mathrm{mmol})$ and tert-butyldimethylsilyl chloride $(6.0 \mathrm{~g}, 40 \mathrm{mmol})$ were added to a solution of the diol in $N, N$-dimethylformamide (DMF) $(60 \mathrm{ml})$. After being stirred at room temperature for $10 \mathrm{~h}$, the reaction was quenched with water $(120 \mathrm{ml})$ and the whole was extracted with EtOAc $(100 \mathrm{ml})$. The organic layer was washed with brine $(2 \times 30 \mathrm{ml})$ and dried over anhydrous $\mathrm{Na}_{2} \mathrm{SO}_{4}$. Filtration and evaporation in vacuo provided the crude product $(16.8 \mathrm{~g})$, which was purified by column chromatography (silica gel $200 \mathrm{~g}, 30: 1$ hexane-EtOAc) to give monosilyl ether $8\left(8.1 \mathrm{~g}, 84 \%\right.$ for 2 steps) as a colorless oil, $[\alpha]_{\mathrm{D}}^{31}-10.0^{\circ}$ $\left(c=1.49\right.$, EtOH). IR (film) $\mathrm{cm}^{-1}: 3472,2959,1464,1256,1088,837 .{ }^{1} \mathrm{H}-$ NMR $\left(\mathrm{CDCl}_{3}\right) \delta: 0.07\left(6 \mathrm{H}, \mathrm{s},\left(\mathrm{CH}_{3}\right)_{2} \mathrm{Si}\right), 0.88\left(3 \mathrm{H}, \mathrm{t}, J=7.4 \mathrm{~Hz}, \mathrm{CH}_{3} \mathrm{CH}_{2}\right)$, $0.90\left(9 \mathrm{H}, \mathrm{s},\left(\mathrm{CH}_{3}\right)_{3} \mathrm{CSi}\right), 0.95\left(3 \mathrm{H}, \mathrm{d}, J=7.0 \mathrm{~Hz}, \mathrm{CH}_{3} \mathrm{CHCH}_{2} \mathrm{O}\right), 0.98(3 \mathrm{H}, \mathrm{d}$, $\left.J=6.6 \mathrm{~Hz}, \mathrm{C}_{3} \mathrm{CH}\right), 1.09\left(1 \mathrm{H}, \mathrm{m}, \mathrm{CH}_{3} \mathrm{CH}_{2}\right), 1.41\left(1 \mathrm{H}, \mathrm{m}, \mathrm{CH}_{3} \mathrm{CH}_{2}\right), 1.50$

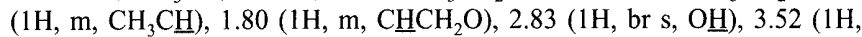
dd, $J=2.5,8.0 \mathrm{~Hz}, \mathrm{C} \underline{\mathrm{HOH}}), 3.68\left(1 \mathrm{H}, \mathrm{dd}, J=4.4,9.8 \mathrm{~Hz}, \mathrm{C}_{2} \mathrm{O}\right), 3.75(1 \mathrm{H}$, dd, $\left.J=3.7,9.8 \mathrm{~Hz}, \mathrm{CH}_{2} \mathrm{O}\right) .{ }^{13} \mathrm{C}-\mathrm{NMR}\left(\mathrm{CDCl}_{3}\right) \delta:-5.84\left(\mathrm{CH}_{3}\right),-5.78$ $\left(\mathrm{CH}_{3}\right), 10.1\left(\mathrm{CH}_{3}\right), 10.8\left(\mathrm{CH}_{3}\right), 14.7\left(\mathrm{CH}_{3}\right), 18.0(\mathrm{C}), 25.5\left(\mathrm{CH}_{2}\right), 25.7$ $\left(\mathrm{CH}_{3}\right), 36.3(\mathrm{CH}), 37.1(\mathrm{CH}), 68.3\left(\mathrm{CH}_{2}\right), 77.5(\mathrm{CH})$. EI-MS $\mathrm{m} / \mathrm{z}$ (rel. int. 
\%): $261\left(\mathrm{M}^{+}+\mathrm{H}, 0.4\right), 243\left(\mathrm{M}^{+}-\mathrm{OH}, 0.4\right), 203(20), 145(6.3), 69$ (100). HR-EI-MS $m / z$ : 243.2148 (Calcd for $\mathrm{C}_{14} \mathrm{H}_{31}$ OSi: 243.2144).

$(2 S, 3 R, 4 S)-3$-[Bis(dimethylamino)phosphoryl]-1-(tert-butyldimethylsilyloxy)-2,4-dimethylhexane (9) To a solution of alcohol 8 (6.88 g, $26 \mathrm{mmol}$ ) in $4: 1 \mathrm{THF}-N, N, N^{\prime}, N^{\prime}$-tetramethylethylenediamine (TMEDA) $(100 \mathrm{ml})$ at $0^{\circ} \mathrm{C}$ was added $n$-butyllithium in hexane $(1.57 \mathrm{M}, 20 \mathrm{ml}, 31$ mmol), followed by tetramethylphosphorodiamidic chloride $(10 \mathrm{ml}, 68$ $\mathrm{mmol}$ ). After being stirred under reflux for $3 \mathrm{~h}$, the reaction was quenched with saturated aqueous $\mathrm{NaHCO}_{3}(40 \mathrm{ml})$. The mixture was diluted with EtOAc $(20 \mathrm{ml})$, and the layers were separated. The aqueous layer was extracted with EtOAc $(40 \mathrm{ml})$, and the combined organic extracts were washed with brine $(2 \times 20 \mathrm{ml})$ and dried over anhydrous $\mathrm{Na}_{2} \mathrm{SO}_{4}$. Filtration and evaporation in vacuo provided the crude product $(13.1 \mathrm{~g})$, which was purified by column chromatography (silica gel $150 \mathrm{~g}, 60: 1 \mathrm{CH}_{2} \mathrm{Cl}_{2}-\mathrm{MeOH}$ ) to give phosphorodiamidate $9(8.29 \mathrm{~g}, 80 \%)$ as a yellow oil, $[\alpha]_{\mathrm{D}}^{29}-2.88^{\circ}(c=1.03$, $\mathrm{CHCl}_{3}$ ). IR (film) $\mathrm{cm}^{-1}: 3358,2930,1462,1304,1211,748 .{ }^{1} \mathrm{H}-\mathrm{NMR}$ $\left(\mathrm{CDCl}_{3}\right) \delta: 0.041\left(3 \mathrm{H}, \mathrm{s},\left(\mathrm{CH}_{3}\right)_{2} \mathrm{Si}\right), 0.044\left(3 \mathrm{H}, \mathrm{s},\left(\mathrm{CH}_{3}\right)_{2} \mathrm{Si}\right), 0.89(9 \mathrm{H}, \mathrm{s}$, $\left.\left(\mathrm{CH}_{3}\right)_{3} \mathrm{CSi}\right), 0.91\left(3 \mathrm{H}, \mathrm{t}, J=7.4 \mathrm{~Hz}, \mathrm{CH}_{3} \mathrm{CH}_{2}\right), 0.93(3 \mathrm{H}, \mathrm{d}, J=6.8 \mathrm{~Hz}$, $\left.\mathrm{CH}_{3} \mathrm{CH}\right), 0.98\left(3 \mathrm{H}, \mathrm{d}, J=6.9 \mathrm{~Hz}, \mathrm{CH}_{3} \mathrm{CHCH}_{2} \mathrm{O}\right), 1.17\left(1 \mathrm{H}, \mathrm{m}, \mathrm{CH}_{3} \underline{\mathrm{H}}_{2}\right)$, $1.58\left(1 \mathrm{H}, \mathrm{m}, \mathrm{CH}_{3} \mathrm{CH}_{2}\right), 1.71\left(1 \mathrm{H}, \mathrm{m}, \mathrm{CH}_{3} \mathrm{C} \underline{\mathrm{H}}\right), 1.91\left(1 \mathrm{H}, \mathrm{m}, \mathrm{C}_{\mathbf{H C H}} \mathrm{O}\right)$, $2.637\left(6 \mathrm{H}, \mathrm{d}, J=9.5 \mathrm{~Hz},\left[\left(\mathrm{CH}_{3}\right)_{2} \mathrm{~N}\right]_{2}\right), 2.641\left(6 \mathrm{H}, \mathrm{d}, J=9.5 \mathrm{~Hz},\left[\left(\mathrm{CH}_{3}\right)_{2} \mathrm{~N}\right]_{2}\right)$, $3.43\left(1 \mathrm{H}, \mathrm{dd}, J=7.3,9.8 \mathrm{~Hz}, \underline{\mathrm{C}}_{2} \mathrm{O}\right), 3.62\left(1 \mathrm{H}, \mathrm{dd}, J=5.7,9.8 \mathrm{~Hz}, \mathrm{CH}_{2} \mathrm{O}\right)$, $4.26(1 \mathrm{H}, \mathrm{dt}, J=9.1,4.1 \mathrm{~Hz}, \mathrm{CHOP}) .{ }^{13} \mathrm{C}-\mathrm{NMR}\left(\mathrm{CDCl}_{3}\right) \delta:-5.35\left(\mathrm{CH}_{3} \times 2\right)$, $12.1\left(\mathrm{CH}_{3}\right), 12.8\left(\mathrm{CH}_{3}\right), 14.8\left(\mathrm{CH}_{3}\right), 18.2(\mathrm{C}), 25.9\left(\mathrm{CH}_{2}, \mathrm{CH}_{3} \times 3\right), 36.7$ $\left(\mathrm{CH}_{3}\right), 38.2\left(J_{\mathrm{C}-\mathrm{P}}=3.7 \mathrm{~Hz}, \mathrm{CH}\right), 38.5\left(J_{\mathrm{C}-\mathrm{P}}=3.7 \mathrm{~Hz}, \mathrm{CH}\right), 66.2\left(\mathrm{CH}_{2}\right), 80.9$ $\left(J_{\mathrm{C}-\mathrm{P}}=3.7 \mathrm{~Hz}, \mathrm{CH}\right)$. FAB-MS $m / z$ (rel. int. \%): $395\left(\mathrm{M}^{+}+\mathrm{H}, 70\right), 153(100)$. HR-FAB-MS $m / z$ : 395.2880 (Calcd for $\mathrm{C}_{18} \mathrm{H}_{44} \mathrm{~N}_{2} \mathrm{O}_{3}$ PSi: 395.2859 ).

$(2 S, 4 S)-2,4-D i m e t h y l h e x a n-1-o l(2)$ Lithium $(760 \mathrm{mg}, 110 \mathrm{mmol})$ was dissolved in ethylamine $(100 \mathrm{ml})$ at $0^{\circ} \mathrm{C}$. After the solution turned blue, a solution of phosphorodiamidate $9(8.19 \mathrm{~g}, 21 \mathrm{mmol})$ in THF $(10 \mathrm{ml})$ was added and the mixture was stirred at $0{ }^{\circ} \mathrm{C}$ for $0.5 \mathrm{~h}$. The reaction was quenched with $\mathrm{NH}_{4} \mathrm{Cl}(1.3 \mathrm{~g})$ and ethylamine was evaporated. The residue was partitioned between $\mathrm{Et}_{2} \mathrm{O}(50 \mathrm{ml})$ and $\mathrm{H}_{2} \mathrm{O}(50 \mathrm{ml})$. The organic layer was successively washed with $1 \mathrm{~N}$ aqueous $\mathrm{HCl}(50 \mathrm{ml})$ and brine $(2 \times 15 \mathrm{ml})$ and dried over anhydrous $\mathrm{Na}_{2} \mathrm{SO}_{4}$. Filtration and evaporation in vacuo afforded the crude product $(4.66 \mathrm{~g})$, which was used without further purification for the next reaction. The silyl ether was dissolved in THF $(20 \mathrm{ml})$ and mixed with $2 \mathrm{~N}$ aqueous $\mathrm{HCl}(20 \mathrm{ml})$. After being stirred for $48 \mathrm{~h}$, the mixture was diluted with $\mathrm{Et}_{2} \mathrm{O}(30 \mathrm{ml})$ and the layers were separated. The organic layer was washed with saturated aqueous $\mathrm{NaHCO}_{3}(10 \mathrm{ml})$ and brine $(2 \times 10 \mathrm{ml})$ and dried over anhydrous $\mathrm{Na}_{2} \mathrm{SO}_{4}$. Filtration and evaporation in vacuo provided the crude product $(5.23 \mathrm{~g})$, which was purified by column chromatography (silica gel $50 \mathrm{~g}, 4: 1$ hexane- $\left.\mathrm{Et}_{2} \mathrm{O}\right)$ to give alcohol $2(2.20 \mathrm{~g}$, $81 \%)$ as a colorless oil, $[\alpha]_{\mathrm{D}}^{27}-4.56^{\circ}\left(c=1.64, \mathrm{CHCl}_{3}\right)$. IR (film) $\mathrm{cm}^{-1}$. $3347,2961,1462,1379,1034 .{ }^{1} \mathrm{H}-\mathrm{NMR}\left(\mathrm{CDCl}_{3}\right) \delta: 0.86(3 \mathrm{H}, \mathrm{t}, J=7.4 \mathrm{~Hz}$, $\left.\mathrm{CH}_{3} \mathrm{CH}_{2}\right), 0.88\left(3 \mathrm{H}, \mathrm{d}, J=6.6 \mathrm{~Hz}, \mathrm{CH}_{3} \mathrm{CH}\right), 0.92\left(3 \mathrm{H}, \mathrm{d}, J=6.6 \mathrm{~Hz}, \mathrm{CH}_{3} \mathrm{CH}\right)$, $1.09(1 \mathrm{H}, \mathrm{m}), 1.28-1.48(4 \mathrm{H}, \mathrm{m}), 1.72(1 \mathrm{H}, \mathrm{m}), 3.38(1 \mathrm{H}, \mathrm{dd}, J=6.8,10.5$ $\left.\mathrm{Hz}, \underline{\mathrm{H}}_{2} \mathrm{OH}\right), 3.51\left(1 \mathrm{H}, \mathrm{dd}, J=5.1,10.5 \mathrm{~Hz}, \mathrm{CH}_{2} \mathrm{OH}\right) .{ }^{13} \mathrm{C}-\mathrm{NMR}\left(\mathrm{CDCl}_{3}\right) \delta$ : $11.2\left(\mathrm{CH}_{3}\right), 17.3\left(\mathrm{CH}_{3}\right), 19.8\left(\mathrm{CH}_{3}\right), 29.0\left(\mathrm{CH}_{2}\right), 31.6(\mathrm{CH}), 33.1(\mathrm{CH}), 40.6$ $\left(\mathrm{CH}_{2}\right), 68.2\left(\mathrm{CH}_{2}\right)$. EI-MS $m / z$ (rel. int. \%): $113\left(\mathrm{M}^{+}-\mathrm{OH}, 15\right), 83(77), 70$ (83), 58 (100). HR-EI-MS $m / z$ : 113.1334 (Calcd for $\mathrm{C}_{8} \mathrm{H}_{17}: 113.1330$ ).

Ethyl (2E,4S,6S)-4,6-dimethyl-2-octenoate (10) A solution of dimethyl sulfoxide (DMSO) $(2.8 \mathrm{ml}, 40 \mathrm{mmol})$ in $\mathrm{CH}_{2} \mathrm{Cl}_{2}(10 \mathrm{ml})$ was added to a solution of oxalyl chloride $(1.6 \mathrm{ml}, 18 \mathrm{mmol})$ in $\mathrm{CH}_{2} \mathrm{Cl}_{2}(40 \mathrm{ml})$ at $-78^{\circ} \mathrm{C}$. After $0.5 \mathrm{~h}$ of stirring at $-78^{\circ} \mathrm{C}$, a solution of alcohol $2(1.95 \mathrm{~g}, 15 \mathrm{mmol})$ in $\mathrm{CH}_{2} \mathrm{Cl}_{2}(10 \mathrm{ml})$ was added. After an additional $0.5 \mathrm{~h}$ of stirring at $-78^{\circ} \mathrm{C}$, triethylamine $(11 \mathrm{ml}, 79 \mathrm{mmol})$ was added. The mixture was stirred at -78 ${ }^{\circ} \mathrm{C}$ for $0.5 \mathrm{~h}$, then was allowed to warm to $0^{\circ} \mathrm{C}$. The reaction was quenched with $\mathrm{H}_{2} \mathrm{O}(30 \mathrm{ml})$ and the mixture was diluted with $10: 1 \mathrm{Et}_{2} \mathrm{O}$-pentane $(70$ $\mathrm{ml}$ ). The layers were separated and the aqueous layer was extracted with 10 : $1 \mathrm{Et}_{2} \mathrm{O}$-pentane $(2 \times 20 \mathrm{ml})$. The combined organic extracts were successively washed with $1 \mathrm{~N}$ aqueous $\mathrm{HCl}(30 \mathrm{ml})$, saturated aqueous $\mathrm{NaHCO}_{3}(20$ $\mathrm{ml}$ ) and brine $(2 \times 10 \mathrm{ml})$, then dried over anhydrous $\mathrm{Na}_{2} \mathrm{SO}_{4}$. Filtration and evaporation in vacuo furnished the crude product $(2.47 \mathrm{~g})$, which was used without further purification for the next reaction. (Carbethoxymethylene)triphenylphos phorane $(6.95 \mathrm{~g}, 20 \mathrm{mmol})$ was added to a solution of the aldehyde in $\mathrm{CH}_{2} \mathrm{Cl}_{2}(20 \mathrm{ml})$. After $5 \mathrm{~h}$ of stirring at room temperature, the solvent was evaporated and the residue $(9.93 \mathrm{~g})$ was purified by column chromatography (silica gel $95 \mathrm{~g}, 40: 1$ hexane- $\mathrm{Et}_{2} \mathrm{O}$ ) to give $(E)-\alpha, \beta$-unsaturated ester $10(2.49 \mathrm{~g}, 84 \%)$ as a colorless oil, $[\alpha]_{\mathrm{D}}^{28}+47.7^{\circ}\left(c=1.13, \mathrm{CHCl}_{3}\right)$. IR (film) $\mathrm{cm}^{-1}: 2963,1723,1651,1462,1370,1273,1182,986 .{ }^{1} \mathrm{H}-\mathrm{NMR}$ $\left(\mathrm{CDCl}_{3}\right) \delta: 0.82-0.87\left(6 \mathrm{H}, \mathrm{m}, \mathrm{CH}_{3} \times 2\right), 1.03\left(3 \mathrm{H}, \mathrm{d}, J=6.7 \mathrm{~Hz}, \mathrm{CH}_{3} \mathrm{CH}\right)$, $1.07-1.17\left(2 \mathrm{H}, \mathrm{m}, \mathrm{CH}_{2}\right), 1.28\left(3 \mathrm{H}, \mathrm{t}, J=7.1 \mathrm{~Hz}, \mathrm{OCH}_{2} \mathrm{CH}_{3}\right), 1.24-1.41$ $\left(3 \mathrm{H}, \mathrm{m}, \mathrm{C} \underline{\mathrm{H}}, \mathrm{C}_{2}\right), 2.40(1 \mathrm{H}, \mathrm{m}, \mathrm{CHCH}=), 4.18(2 \mathrm{H}, \mathrm{q}, J=7.1 \mathrm{~Hz}$,
$\left.\mathrm{OCH}_{2} \mathrm{CH}_{3}\right), 5.77(1 \mathrm{H}, \mathrm{d}, J=15.7 \mathrm{~Hz},=\mathrm{CHCO}), 6.81(1 \mathrm{H}, \mathrm{dd}, J=8.4,15.7$ $\mathrm{Hz}, \mathrm{CHC} \underline{\mathrm{H}}=) .{ }^{13} \mathrm{C}-\mathrm{NMR}\left(\mathrm{CDCl}_{3}\right) \delta: 11.1\left(\mathrm{CH}_{3}\right), 14.3\left(\mathrm{CH}_{3}\right), 18.9\left(\mathrm{CH}_{3}\right)$, $20.4\left(\mathrm{CH}_{3}\right), 29.8\left(\mathrm{CH}_{2}\right), 31.9(\mathrm{CH}), 34.3(\mathrm{CH}), 43.4\left(\mathrm{CH}_{2}\right), 60.1\left(\mathrm{CH}_{2}\right)$, $119.6(\mathrm{CH}), 154.7(\mathrm{CH}), 166.9(\mathrm{C}=\mathrm{O})$. EI-MS $m / z$ (rel. int. \%): $198\left(\mathrm{M}^{+}\right.$, 2.6), 169 (30), 153 (30), 141 (44), 69 (100). HR-EI-MS m/z: 198.1626 (Calcd for $\mathrm{C}_{12} \mathrm{H}_{22} \mathrm{O}_{2}: 198.1620$ ).

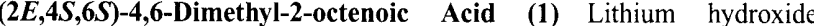
monohydrate $(1.28 \mathrm{~g}, 31 \mathrm{mmol})$ was added to a solution of ester $\mathbf{1 0}(1.98 \mathrm{~g}$, $10.0 \mathrm{mmol})$ in $3: 1 \mathrm{MeOH}-\mathrm{H}_{2} \mathrm{O}(20 \mathrm{ml})$ at room temperature. After $12 \mathrm{~h}$ of stirring at room temperature, the solvent was evaporated in vacuo. The residue was dissolved in $\mathrm{H}_{2} \mathrm{O}(15 \mathrm{ml})$ and the whole was washed with hexane $(2 \times 10 \mathrm{ml})$. The aqueous layer was acidified with $10 \%$ aqueous $\mathrm{HCl}$ $(20 \mathrm{ml})$, and was extracted with EtOAc $(20 \mathrm{ml})$. The organic layer was washed with brine $(2 \times 5 \mathrm{ml})$ and dried over anhydrous $\mathrm{Na}_{2} \mathrm{SO}_{4}$. Filtration and evaporation in vacuo, followed by bulb-to-bulb distillation, afforded carboxylic acid $1(1.67 \mathrm{~g}, 98 \%)$ as a colorless oil, bp $120^{\circ} \mathrm{C}(1 \mathrm{mmHg}),[\alpha]_{\mathrm{D}}^{24}$ $+58.6^{\circ}$ (neat, $d^{24}=0.954$ ) [lit. ${ }^{27)}[\alpha]_{\mathrm{D}}^{19}+55^{\circ}$ (neat)]. IR (film) $\mathrm{cm}^{-1}: 2963$, 1698, 1420, 1287, 988. 'H-NMR $\left(\mathrm{CDCl}_{3}\right) \delta: 0.83-0.88\left(6 \mathrm{H}, \mathrm{m}, \mathrm{CH}_{3} \mathrm{CH}_{2}\right.$, $\left.\mathrm{CH}_{3} \mathrm{CH}\right), 1.05\left(3 \mathrm{H}, \mathrm{d}, J=6.7 \mathrm{~Hz}, \mathrm{CH}_{3} \mathrm{CH}\right), 1.09-1.18(2 \mathrm{H}, \mathrm{m}), 1.25-1.42$ $(3 \mathrm{H}, \mathrm{m}), 2.45(1 \mathrm{H}, \mathrm{m}, \mathrm{C} \underline{\mathrm{HCH}}=), 5.79\left(1 \mathrm{H}, \mathrm{d}, J=15.6 \mathrm{~Hz},=\mathrm{CHCO}_{2} \mathrm{H}\right), 6.94$ $(1 \mathrm{H}, \mathrm{dd}, J=8.4,15.6 \mathrm{~Hz}, \mathrm{CHCH}=) \cdot{ }^{13} \mathrm{C}-\mathrm{NMR}\left(\mathrm{CDCl}_{3}\right) \delta: 11.2\left(\mathrm{CH}_{3}\right), 18.8$ $\left(\mathrm{CH}_{3}\right), 20.2\left(\mathrm{CH}_{3}\right), 29.8\left(\mathrm{CH}_{2}\right), 31.9(\mathrm{CH}), 34.4(\mathrm{CH}), 43.3\left(\mathrm{CH}_{2}\right), 119.0$ $(\mathrm{CH}), 157.3(\mathrm{CH}), 172.4(\mathrm{C}=\mathrm{O})$. EI-MS $\mathrm{m} / z$ (rel. int. \%): $170\left(\mathrm{M}^{+}, 2.5\right)$, 155 (2.0), 141 (35), 114 (34), 100 (68), 43 (100). HR-EI-MS m/z: 170.1314 (Calcd for $\mathrm{C}_{10} \mathrm{H}_{18} \mathrm{O}_{2}: 170,1306$ )

Acknowledgments This research was supported in part by a Grant-inAid for Scientific Research on Priority Areas from the Ministry of Education, Science, Sports and Culture, Japan.

\section{References and Notes}

1) a) Biller S. A., Neuenschwander K., Ponpipom M. M., Poulter C. D., Curr. Pharm. Des., 2, $1-40$ (1996); b) Watson N. S., Procopiou P. A., "Progress in Medicinal Chemistry," Vol. 33, ed. by Ellis G. P., Luscombe D. K., Elsevier Science, 1996, pp. 331-378 and references cited therein.

2) For reviews, see: a) Koert U., Angew. Chem., Int. Ed. Engl., 34, 773778 (1995); b) Nadin A., Nicolaou K. C., Angew. Chem., Int. Ed. Engl., 35, 1622-1656 (1996).

3) For recent synthetic studies, see: $a$ ) Kataoka O., Kitagaki S., Watanabe N., Kobayashi J., Nakamura S., Shiro M., Hashimoto S., Tetrahedron Lett., 39, 2371-2374 (1998); b) Reid A. M., Steel P. G., J. Chem. Soc., Perkin Trans. 1, 1998, 2795-2801; c) Fraisse P., Hanna I., Lallemand J.-Y., Tetrahedron Lett., 39, 7853 -7856 (1998); d) Martin S. F., Naito S., J. Org. Chem., 63, 7592-7593 (1998); e) Freeman-Cook K. D., Halcomb R. L., Tetrahedron Lett., 39, 8567-8570 (1998); f) Tsubuki M., Okita H., Honda T., Synlett, 1998, 1417-1419; g) Koshimizu H., Baba T., Yoshimitsu T., Nagaoka H., Tetrahedron Lett., 40, 2777-2780 (1999).

4) Nicolaou K. C., Yue E. W., La Greca S., Nadin A., Yang Z., Leresche J. E., Tsuri T., Naniwa Y., De Riccardis F., Chem. Eur. J., 1, 467_-494 (1995).

5) a) Stoermer D., Caron S., Heathcock C. H., J. Org. Chem., 61, 91159125 (1996); b) Caron S., Stoermer D., Mapp A. K., Heathcock C. H., J. Org. Chem., 61, 9126-9134 (1996).

6) Carreira E. M., Du Bois J., J. Am. Chem. Soc., 117, 8106-8125 (1995).

7) Evans D. A., Barrow J. C., Leighton J. L., Robichaud A. J., Sefkow M., J. Am. Chem. Soc., 116, $12111-12112$ (1994).

8) Armstrong A., Jones L. H., Barsanti P. A., Tetrahedron Lett., 39, 3337-3340 (1998)

9) Sato H., Nakamura S., Watanabe N., Hashimoto S., Synlett, 1997, $451-454$.

10) a) Schlessinger R. H., Gillman K. W., Tetrahedron Lett., 37, 13311334 (1996); b) Tomooka K., Nagasawa A., Wei S.-Y., Nakai T., Tetrahedron Lett., 37, 8895-8898 (1996).

11) Evans D. A., Ennis M. D., Mathre D. J., J. Am. Chem. Soc., 104, $1737-1739$ (1982).

12) Evans D. A., Dow R. L., Shih T. L., Takacs J. M., Zahler R., J. Am. Chem. Soc., 112, 5290-5313 (1990).

13) Decicco C. P., Grover P., J. Org. Chem., 61, 3534-3541 (1996).

14) a) Evans D. A., Takacs J. M., Tetrahedron Lett., 21, 4233-4236 (1980); b) Sonnet P. E., Heath R. R., J. Org. Chem., 45, 3137-3139 (1980). 
15) White J. D., Johnson A. T., J. Org. Chem., 59, 3347-3358 (1994),

16) Birkbeck A. A., Enders D., Tetrahedron Lett., 39, 7823-7826 (1998).

17) Highly efficient methods to skip 1,3-dimethyl motif via auxiliarybased alkylation have very recently been developed. a) Abiko A., Masamune S., Tetrahedron Lett., 37, 1081-1084 (1996); b) Myers A. G., Yang B. H., Chen H., McKinstry L., Kopecky D. J., Gleason J. L., J. Am. Chem. Soc., 119, 6496-6511 (1997).

18) a) Evans D. A., Bartroli J., Shih T. L., J. Am. Chem. Soc., 103, 2127$2129(1981)$; b) Gage J. R., Evans D. A., Org. Synth., 68, 83-91 (1989).

19) White J. D., Bolton G. L., Dantanarayana A. P., Fox C. M. J., Hiner R. N., Jackson R. W., Sakuma K., Warrier U. S., J. Am. Chem. Soc., 117, 1908-1939 (1995).

20) A similar approach to skip 1,3-dimethyl arrays based on the combinational use of Evans aldol reaction and Barton-McCombie deoxygenation has been adopted by Yamada and co-workers, culminating in a total synthesis of doliculide. Ishiwata H., Sone H., Kigoshi H., Yamada K., Tetrahedron, 50, 12853-12882 (1994).

21) Evans D. A., Bartroli J., Tetrahedron Lett., 23, 807-810 (1982).

22) Penning T. D., Djuric' S. W., Haack R. A., Kalish V. J., Miyashiro J. M., Rowell B. W., Yu S. S., Synth. Commun., 20, 307-312 (1990).

23) McCombie S. W., "Comprehensive Organic Synthesis," Vol. 8, ed. by Trost B. M., Fleming I., Pergamon Press, Oxford, 1991, pp. 811-833.

24) Hanessian S., Murray P. J., Tetrahedron, 43, 5055-5072 (1987).

25) Ireland R. E., Muchmore D. C., Hengartner U., J. Am. Chem. Soc., 94, 5098-5100 (1972)

26) Barton D. H. R., McCombie S. W., J. Chem. Soc., Perkin Trans. I, 1975, 1574-1585.

27) Sidebottom P. J., Highcock R. M., Lane S. J., Procopiou P. A., Watson N. S., J. Antibiot., 45, 648-658 (1992).

28) Inoue T., Mukaiyama T., Bull. Chem. Soc. Jpn., 53, 174-178 (1980). 